\title{
Large-Scale Albuminuria Screen for Nephropathy Models in Chemically Induced Mouse Mutants
}

\author{
Birgit Rathkolb ${ }^{a}$ Tuyen V. Tran ${ }^{a}$ Martina Klempt ${ }^{a}$ Martin Hrabé de Angelis ${ }^{b}$ \\ Rüdiger Wanke ${ }^{c}$ Eckhard Wolf ${ }^{a}$ Bernhard Aigner ${ }^{a}$ \\ ${ }^{a}$ Institute of Molecular Animal Breeding and Biotechnology, Ludwig Maximilians University, Munich; \\ ${ }^{b}$ Institute of Experimental Genetics, GSF Research Center for Environment and Health, Neuherberg, and \\ ${ }^{\mathrm{c}}$ Institute of Veterinary Pathology, Ludwig Maximilians University, Munich, Germany
}

\section{Key Words}

Albumin · Ethylnitrosourea $\cdot$ Kidney lesions •

Proteinuria

\begin{abstract}
Background/Aim: Phenotype-driven screening of a great pool of randomly mutant mice and subsequent selection of animals showing symptoms equivalent to human kidney diseases may result in the generation of novel suitable models for the study of the pathomechanisms and the identification of genes involved in kidney dysfunction. Methods: We carried out a large-scale analysis of ethylnitrosourea (ENU)-induced mouse mutants for albuminuria by using qualitative SDS-polyacrylamide gel electrophoresis. Results: The primary albuminuria screen preceded the comprehensive phenotypic mutation analysis in a part of the mice of the Munich ENU project to avoid loss of mutant animals as a consequence of prolonged suffering from severe nephropathy. The primary screen detected six confirmed phenotypic variants in 2,011 G1 animals screened for dominant mutations and no variant in $48 \mathrm{G} 3$ pedigrees screened for recessive mutations. Further breeding experiments resulted in two lines showing a low phenotypic penetrance of albuminuria. The secondary albuminuria screen was carried out in mutant lines which were established in the
\end{abstract}

Munich ENU project without preceding primary albuminuria analysis. Two lines showing increased plasma urea levels were chosen to clarify if severe kidney lesions are involved in the abnormal phenotype. This analysis revealed severe albuminuria in mice which are affected by a recessive mutation leading to increased plasma urea and cholesterol levels. Conclusion: Thus, the phenotypic selection of ENU-induced mutants according to the parameter proteinuria in principle demonstrates the feasibility to identify nephropathy phenotypes in ENUmutagenized mice.

Copyright $(2005$ S. Karger AG, Basel

Research on human diseases is often hindered by the lack of suitable laboratory animal models. Genetic engineering techniques allow the defined alteration of the mouse genome, however, the resulting phenotype of the mutant mice cannot be predicted. A complementary strategy for the establishment of suitable animal models for human diseases consists of the production of a great pool of mutant mice exhibiting different phenotypes and the subsequent selection of relevant lines according to similarities in the pathologic phenotype of animal model and human patients [1]. Forward genetics techniques subsequently identify the causative mutation in the lines [2].

\section{KARGER}

Fax +4161306 1234 E-Mail karger@karger.ch www.karger.com
(C) 2005 S. Karger AG, Basel $1660-2129 / 05 / 1004-0143 \$ 22.00 / 0$

Accessible online at: www.karger.com/nee
Bernhard Aigner, Institut für Molekulare Tierzucht und Biotechnologie

Moorversuchsgut, Hackerstrasse 27

DE-85764 Oberschleissheim (Germany)

Tel. +4989218078420, Fax +4989218078422

E-Mail b.aigner@gen.vetmed.uni-muenchen.de 
$\mathrm{N}$-ethyl-N-nitrosourea (ENU) has been used in various mouse mutagenesis programs to produce random mutations. Specific pathologic phenotypes were identified in the offspring of the ENU-treated mice by the use of appropriate routine procedures allowing the screening of large numbers of mice for a broad spectrum of parameters. Successful detection of the causative mutations in the phenotypically altered mice has been demonstrated in already examined ENU-induced mutant mouse lines. Both ethical and economic considerations on the large number of animals involved in the ENU projects force the implementation of a high number of different phenotypic screens to detect as many mutants as possible $[1,3]$.

In the Munich ENU Mouse Mutagenesis Project, the animals are examined in various phenotypic screens to characterize mouse mutants with defects of diverse organ systems or changes in metabolic pathways [4]. A screening profile of clinical chemistry parameters was established to detect deviations of normal blood substrate and electrolyte levels or enzyme activities. Transmission of the altered phenotype to the subsequent generations was confirmed by breeding of the affected mice and the screening of the offspring, thereby revealing a mutation as cause for the aberrant phenotype $[5,6]$.

Nephropathies include various multifactorial disorders caused by genetic and/or environmental factors. Several monogenic disorders are known to result in progressive renal insufficiency. Among these are glomerular basement membrane defects as well as tubular and interstitial alterations. Once chronic renal insufficiency is established, it tends to progress to end-stage kidney failure, which is characterized by progressive glomerulosclerosis irrespective of the initiating nephropathy [7].

In glomerular diseases, proteinuria appears as a consequence of the pathologic transglomerular passage of high-molecular-weight (HMW) proteins due to the increased permeability of the glomerular capillary wall and the impaired reabsorption by the epithelial cells in the proximal tubuli. The load of these proteins in the tubular lumen leads to the saturation of the reabsorptive mechanism of the tubular cells. This results in the urinary excretion of proteins including the low-molecular-weight (LMW) proteins, which are reabsorbed under physiologic conditions. Thus, proteinuria is used as a marker for the course as well as for the response to treatments in patients with glomerular diseases [8]. In addition, proteinuria acts as an independent risk factor for the progression of renal failure $[9,10]$.
In humans, quantitative as well as qualitative examination of urinary protein excretion is suitable to detect early kidney lesions [8]. Mice physiologically excrete large amounts of LMW proteins with the urine (major urinary proteins, MUPs) [11]. Therefore, a qualitative screen for albumin, an intermediate-molecular-weight (IMW) protein $(\sim 70 \mathrm{kDa})[12]$, was chosen to identify mutant mice which might represent novel nephropathy models. This study describes the identification of albuminuric mouse mutants by screening a large number of ENU-mutagenized mice as well as the intrinsic problems associated with this approach.

\section{Materials and Methods}

\section{Mutagenesis and Breeding of Mice}

In the Munich ENU Mouse Mutagenesis Project, 10-week-old inbred male $\mathrm{C} 3 \mathrm{HeB} / \mathrm{FeJ}(\mathrm{C} 3 \mathrm{H})$ mice were injected intraperitoneally with ENU $(3 \times 90 \mathrm{mg} / \mathrm{kg}$ in weekly intervals). The screen for dominant mutations was performed on $\mathrm{G} 1$ animals derived from mutagenized $\mathrm{C} 3 \mathrm{H}$ males mated to wild-type $\mathrm{C} 3 \mathrm{H}$ females. Inheritance of the observed abnormal phenotype in the inbred $\mathrm{C} 3 \mathrm{H}$ genetic background was tested in $\mathrm{G} 2$ offspring from the mating of the affected G1 mouse exhibiting the altered phenotype and wild-type mice. The screen for recessive mutations was carried out on G3 mice produced in a two-step breeding scheme from G1 males. G1 males which were excluded to harbor dominant mutations in the comprehensive phenotypic mutation analysis of the Munich ENU project including the albuminuria analysis and the clinical chemistry screen were mated to wild-type $\mathrm{C} 3 \mathrm{H}$ females for the production of G2 offspring. Subsequently, 6-8 G2 females were backcrossed to the G1 male to produce the G3 offspring of the pedigree which were analyzed for recessive mutations. Inheritance of the observed abnormal phenotype in the inbred $\mathrm{C} 3 \mathrm{H}$ genetic background was tested on $\mathrm{G} 4$ intercross or $\mathrm{G} 4 \times \mathrm{G} 3$ backcross offspring after breeding the $\mathrm{G} 3$ phenotypic variants to wild-type mice. After the identification of the causative mutation, the mutant lines will be designated according to the official nomenclature. Mouse husbandry was done under a continuously controlled specific pathogen-free hygiene standard according to the FELASA protocols [http://www. felasa.org]. Standard diet and water were provided ad libitum. All animal experiments were carried out under the approval of the responsible animal welfare authority.

\section{Examination of Albuminuria}

Examination of albuminuria in G1 and G3 offspring of ENUtreated $\mathrm{C} 3 \mathrm{H}$ mice was carried out by the qualitative analysis of urinary proteins using SDS-polyacrylamide gel electrophoresis (SDS-PAGE). 20- $\mu$ l spot urine samples were taken from the mice 2 months post-partum and stored at $-20^{\circ} \mathrm{C}$. Urine samples were boiled for 10 min after 1:2 dilution with sample buffer $(62.5 \mathrm{mM}$ Tris- $\mathrm{HCl}$ pH 6.8, 2\% SDS, 25\% glycerol, $0.01 \%$ bromophenol blue, $5 \%$ 2-mercaptoethanol). $10 \mu \mathrm{l}$ of the samples were electrophoresed (25 m $M$ Tris, $200 \mathrm{~m} M$ glycine, $0.1 \%$ SDS) in a Tris-HCl-polyacrylamide gel (4-20\% gradient) using the Bio-Rad Mini Protean II system (Bio-Rad Laboratories, Hercules, Calif., USA). Protein bands 
were visualized by staining with Coomassie Brilliant Blue. The broad range SDS-PAGE standard (Bio-Rad Laboratories) was used as molecular weight standard for the detected bands. Wild-type $\mathrm{C} 3 \mathrm{H}$ mice were used as negative controls. Stained gels were photographed and dried for documentation.

\section{Evaluation of the SDS-PAGE Analysis for the Detection of Albuminuria}

In mice, urine proteins indicating early glomerular lesions (albumin), advanced glomerular lesions (HMW proteins) and tubular lesions (proteins ranging between MUPs and albumin) were identified by SDS-PAGE [13]. The evaluation of the appropriate urine protein detection method allowing the screening of a large number of animals was carried out using growth hormone transgenic mice which were described to inevitably develop progressive kidney lesions including glomerulosclerosis with secondary tubulointerstitial lesions [14]. SDS-PAGE urine analysis of growth hormone transgenic mice of two different lines and their control littermates resulted in the consistent detection of the development of severe albumin excretion in the transgenic animals. The identification of albumin was carried out by the comparison to molecular weight standards. In addition, albuminuria was confirmed by Western blot analysis using rabbit anti-mouse albumin antibody (Biotrend, Cologne, Germany) and horseradish peroxidase-conjugated swine anti-rabbit antibody (Dako Diagnostika, Hamburg, Germany). Wild-type littermates were used as negative controls.

\section{Clinical Chemistry Screen}

Blood samples from 3-month-old G1 and G3 mice fasted overnight were obtained by puncture of the retroorbital sinus under ether anesthesia. Physiologic parameter values were determined in male and female $\mathrm{C} 3 \mathrm{H}$ controls. Plasma from Li-heparin-treated blood was analyzed using an Olympus AU400 autoanalyser (Olympus, Hamburg, Germany) and the adapted reagents (Olympus). Calibration and quality control were performed according to the manufacturer's protocols. The clinical chemistry screen included the following plasma parameters: substrates: creatinine, ferritin, glucose, total protein, total cholesterol, transferrin, triglycerides, urea, uric acid; electrolytes: calcium, chloride, iron, phosphorus, potassium, sodium; enzymes: alanine aminotransferase, alkaline phosphatase, amylase, aspartate aminotransferase, creatine kinase, lactate dehydrogenase, lipase.

\section{Results}

\section{Primary Screen for Albuminuria in ENU-Induced \\ Mouse Mutants}

The primary albuminuria screen preceded the comprehensive phenotypic mutation analysis in a part of the mice of the Munich ENU Mouse Mutagenesis Project to avoid loss of mutant animals as a consequence of prolonged suffering from severe nephropathy. The search for mutant mice showing albuminuria was carried out on the inbred $\mathrm{C} 3 \mathrm{H}$ genetic background 2 months post-partum. Spot urine samples were collected in the morning and analyzed by SDS-PAGE. The animals positive for albu-
Table 1. Albuminuria screen in ENU-induced mouse mutants

\begin{tabular}{lllll}
\hline Screen & $\mathrm{n}(\mathrm{m} / \mathrm{f})^{\mathrm{a}}$ & Pedigrees $^{\mathrm{b}}$ & $\begin{array}{l}\text { Variants }^{\mathrm{c}} \\
(\mathrm{m} / \mathrm{f})\end{array}$ & $\begin{array}{l}\text { Mutants }^{\mathrm{d}} \\
(\mathrm{m} / \mathrm{f})\end{array}$ \\
\hline Dominant (G1) & $\begin{array}{l}2,011 \\
(1,200 / 811)\end{array}$ & 317 & $6(5 / 1)$ & $2(2 / 0)$ \\
Recessive (G3) & $\begin{array}{l}1,016 \\
(518 / 498)\end{array}$ & 48 & 0 & - \\
\hline Total & 3,027 & 365 & 6 & 2 \\
\hline
\end{tabular}

${ }^{a} \mathrm{~m} / \mathrm{f}$, males/females.

${ }^{\mathrm{b}}$ Corresponds to the number of ENU-treated founder mice used for the breeding of the G1 and G3 animals.

${ }^{\mathrm{c}}$ Offspring of ENU-treated mice showing albuminuria in both tests in a 2-week interval.

${ }^{\mathrm{d}}$ Variants inheriting albuminuria to subsequent generations.

minuria were retested in a second analysis 2 weeks later to confirm the abnormal phenotype.

In the nearly 3 years' examination interval $(04 / 01-$ 01/04), urine samples from 3,027 animals were analyzed. For the search of dominant mutations, 2,011 G1 offspring of ENU-treated mice were screened. Nine animals were found to be positive in the first analysis and 6 of them $(0.3 \%$ of the analyzed mice) were confirmed in the second analysis (table 1). One animal showing strong albuminuria died after the first test. The other 2 mice were negative in the second analysis and, therefore, were assumed to harbor no mutation. Continuous albuminuria was found in the six confirmed phenotypic variants by observing the abnormal phenotype also in urine samples taken before the euthanasia of the animals at different ages between 6 and 20 months post-partum (fig. 1). The 6 mice with confirmed albuminuria were derived from 5 different mutagenized founders.

To avoid additional damage of the general condition of the six G1 phenotypic variants, we refrained from the recovery of blood for the comprehensive clinical chemistry analysis, but directly set up the confirmation crosses by mating the affected mice to wild-type animals. Four variants produced no positive offspring, whereas two lines were established which transmitted the albuminuric phenotype to the subsequent generations. 50\% phenotypic penetrance of the symptom was expected for dominant mutations in our breeding scheme. However, in both lines only 4 of 63 G2 offspring (6.4\%) showed albuminuria. The low penetrance was also observed in the two subsequent generations examined in the line $32011 \mathrm{~m}(4.5 \%$ overall penetrance) (table 2). In non-albuminuric off- 


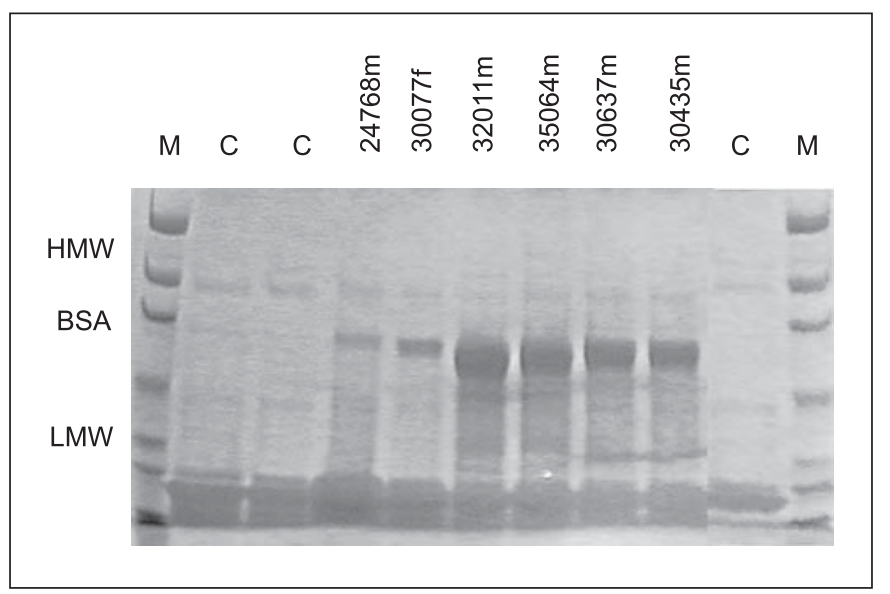

Fig. 1. SDS-PAGE albuminuria analysis of G1 offspring of ENUtreated mice. Protein bands were visualized by staining with Coomassie Brilliant Blue. The broad range SDS-PAGE standard (BioRad Laboratories) was used as molecular weight standard (M) for the identification of the albumin excretion: HMW = high-molecular-weight proteins; BSA = bovine serum albumin; LMW = lowmolecular-weight proteins. The positive analysis of the urine taken before the euthanasia of the six confirmed phenotypic variants is shown, thereby revealing the continuous albuminuria from the onset 2 months post-partum until the euthanasia of the mice. Age of the animals in months (mo): $24768 \mathrm{~m}, 18 \mathrm{mo} ; 30077 \mathrm{f}, 17 \mathrm{mo}$; $30435 \mathrm{~m}, 6 \mathrm{mo} ; 30637 \mathrm{~m}, 10 \mathrm{mo} ; 32011 \mathrm{~m}, 16 \mathrm{mo} ; 35064 \mathrm{~m}, 20 \mathrm{mo}$. $\mathrm{m}=$ Male; $\mathrm{f}=$ female. Wild-type $\mathrm{C} 3 \mathrm{H}$ samples of various age were used as negative controls (C).

Table 2. Inheritance analysis of albuminuria in the offspring of the dominant phenotypic variants

\begin{tabular}{llll}
\hline Variant & Mutant $^{\mathrm{a}}$ & $\begin{array}{l}\text { Offspring tested }^{\mathrm{b}} \\
\mathrm{n}(\mathrm{m} / \mathrm{f})\end{array}$ & $\begin{array}{l}\text { Albuminuria } \\
\mathrm{n}(\mathrm{ID})^{\mathrm{c}}\end{array}$ \\
\hline $24768 \mathrm{~m}$ & & $28(13 / 15)$ & 0 \\
$30077 \mathrm{f}$ & & $22(11 / 11)$ & 0 \\
$30435 \mathrm{~m}$ & & $10(6 / 4)$ & 0 \\
$30637 \mathrm{~m}$ & & $8(4 / 4)$ & 0 \\
$32011 \mathrm{~m}$ & & $30(17 / 13)$ & $2(37226 \mathrm{f}, 37324 \mathrm{~m})$ \\
& $37226 \mathrm{f}$ & $13(9 / 4)$ & $1(36198 \mathrm{~m})$ \\
& $37324 \mathrm{~m}$ & $10(5 / 5)$ & $1(43006 \mathrm{~m})$ \\
& $36198 \mathrm{~m}$ & $24(15 / 9)$ & 0 \\
$35064 \mathrm{~m}$ & $43006 \mathrm{~m}$ & $20(9 / 11)$ & $1(49909 \mathrm{~m})$ \\
& & $33(17 / 16)$ & $2(43356 \mathrm{f}, 48442 \mathrm{f})$ \\
\hline
\end{tabular}

a Animal number of the G1 offspring of ENU-treated mice showing albuminuria in both tests in a 2-week interval (phenotypic variants) and the mice inheriting the albuminuria (mutants). $\mathrm{m} / \mathrm{f}=$ Males/females. The two positive mice $30435 \mathrm{~m}$ and $30637 \mathrm{~m}$ were offspring of the same founder.

${ }^{\mathrm{b}}$ The offspring were produced by mating the affected mice (variants/mutants) to wild-type animals.

${ }^{\mathrm{c}}$ ID = animal number of the positive mice. spring of the two mutant lines (line $32011 \mathrm{~m}: \mathrm{n}=16$; line $35064 \mathrm{~m}: \mathrm{n}=7$ ), clinical chemistry blood analysis was carried out for the search of a correlated phenotypic marker to detect additional mutant individuals, but no conspicuous deviations were observed.

Six offspring per litter were obtained on average 3 weeks post-partum in the matings of both the two mutants and the four phenotypic variants. Comparable numbers were detected in the matings of wild-type $\mathrm{C} 3 \mathrm{H}$ mice. Deviations in the sex ratio of the offspring were also not observed. In addition, an impaired general condition leading to a decreased lifetime was revealed only in 4 of the 14 animals exhibiting albuminuria. In total, early loss of mutants before the examination time point may occur to a certain degree, but can explain the low phenotypic penetrance only to a minor part.

For revealing recessive mutations, 1,016 G3 offspring were examined which were derived from 48 pedigrees. None was found to be positive in the first analysis 2 months post-partum.

\section{Secondary Albuminuria Screen in Established ENU Mutant Lines}

In the Munich ENU Mouse Mutagenesis Project, the clinical chemistry analysis of more than $15,000 \mathrm{G1}$ mice and $230 \mathrm{G} 3$ pedigrees resulted in the establishment of mutant lines due to various blood deviations [http://www. lmb.uni-muenchen.de/groups/mt/mainmt2.htm; Rathkolb et al., unpubl. results]. Two mutant lines established in the project without preceding primary albuminuria analysis which showed increased plasma urea levels were chosen for the secondary albuminuria screen to clarify if severe kidney lesions are involved in the abnormal phenotype of these lines.

Line $10068383 \mathrm{~m}$ harboring a recessive mutation was established due to increased plasma urea $(75-100 \mathrm{mg} / \mathrm{dl})$ and cholesterol (190-300 mg/dl) levels of the affected homozygous mutant mice. In addition, the animals showed a decreased lifetime of about 5 months. Unaffected heterozygous mutant and wild-type littermates as well as $\mathrm{C} 3 \mathrm{H}$ controls showed physiologic plasma levels of urea $(25-70 \mathrm{mg} / \mathrm{dl})$ and cholesterol $(75-160 \mathrm{mg} / \mathrm{dl})$. Heterozygous mutant and wild-type littermates also were not distinguishable in the other parameters of the clinical chemistry blood analysis. After breeding heterozygous mutant mice of the line $10068383 \mathrm{~m}$, the urine protein analysis of the offspring revealed severe albumin excretion in the homozygous mutants (fig. 2). The results of the albuminuria screen were confirmed by the preliminary pathohistologic examination of kidney samples of the homozy- 
Fig. 2. SDS-PAGE albuminuria analysis of line $10068383 \mathrm{~m}$ harboring a recessive mutation. Protein bands were visualized by staining with Coomassie Brilliant Blue. The broad range SDSPAGE standard (Bio-Rad Laboratories) was used as molecular weight standard $(M)$ for the identification of the albumin excretion: HMW = high-molecular-weight proteins; BSA = bovine serum albumin; LMW = low-molecular-weight proteins. Offspring of heterozygous mutants were examined and severe albuminuria was observed to be strictly correlated to the homozygous mutant mice showing increased plasma urea and cholesterol levels (HO). Dilutions of the urine of 4-week-old mice were used for the preparation of the figure. Older homozygous mutants of this line showed severely increased albuminuria. $\mathrm{W}=$ Heterozygous mutant or wildtype littermates showing normal plasma urea and cholesterol levels.

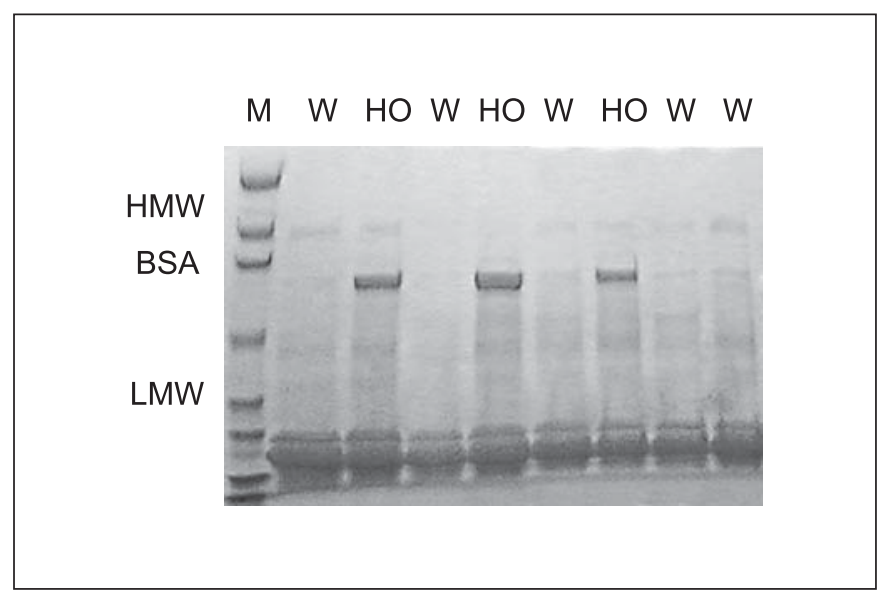

Fig. 3. Histopathologic kidney analysis of homozygous mutant mice of the line $10068383 \mathrm{~m}$ harboring a recessive mutation. Light microscopic aspect of kidney changes in an 11-week-old female homozygous mutant. Severe glomerular lesions with obliteration of glomerular capillaries and proteinaceous casts in tubular lumina are present. Paraffin section, hematoxylin and eosin stain, scale bar $50 \mu \mathrm{m}$. Subsequent analyses have to be carried out to reveal the detailed mutant renal phenotype of the line.

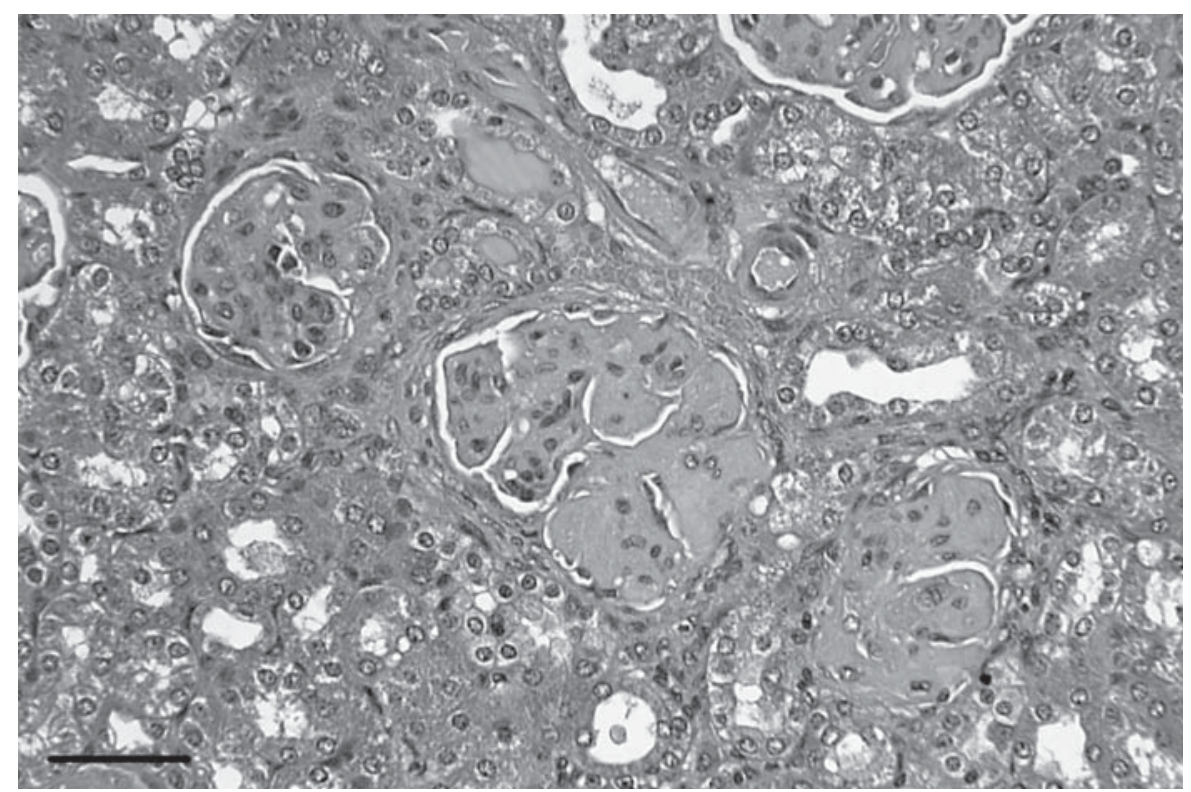

gous mutant mice where severe alterations were observed (fig. 3).

Heterozygous mutant mice of a second line $(20003171 \mathrm{~m})$ showed increased plasma urea levels analogous to line $10068383 \mathrm{~m}$ due to a dominant mutation. In addition, the clinical chemistry blood analysis of the mutants showed significant deviations in the parameters glucose, total protein, triglycerides, alkaline phosphatase and amylase. No albuminuria was observed in the mutant mice (14 heterozygous mutants) in the secondary albuminuria screen. The pathohistologic analysis of the kidney samples also showed no gross alterations (not shown).

Albuminuria Screen in ENU Mutants

\section{Discussion}

Qualitative SDS-PAGE analysis of urinary proteins resulted in the identification of ENU-induced mouse mutants showing the pathologic excretion of serum albumin. Transmission of the deviation to the subsequent generations led to the establishment of lines showing albuminuria.

Reproduction of the positive primary results in the subsequent tests succeeded in most of the phenotypic variants as well as in the examined positive offspring in the lines showing albuminuria. Failure of the transmission of the defect to the offspring in four of the six mated phenotypic variants was also found in a comparable ratio 
for the inheritance of other phenotypic deviations in our clinical chemistry screen [Rathkolb et al., unpubl. results]. One reason may be the occurrence of not genetically fixed proteinuria due to the inherent impossibility of carrying out complete standardization of husbandry and experiment. In addition, the urine of normal mice contains small amounts of albumin [12]. Faint signals of the same molecular weight were inconsistently seen in unaffected mice of our study in the SDS-PAGE analysis. Urine albumin content in normal mice was estimated to correspond to $0.5-1.5 \mathrm{mg} / \mathrm{dl}$ by subsequent clinical chemistry urine analysis. Thus, the pathologic increase of the normally low albumin excretion was used as phenotypic marker.

A low phenotypic penetrance of the defect was revealed in both mutant lines where $50 \%$ of the offspring in our breeding scheme were expected to show albuminuria. Appearance of albuminuria due to the interaction of multiple mutations leads to lower levels of affected offspring. In addition, variations in the concentration of the urine samples might influence the outcome of the screen by the failure in the detection of albuminuria which may be observed by measuring the albumin/creatinine ratio. Alternatively, the incomplete penetrance might be due to the analysis of mice at an early age. The early examination time point was chosen to avoid loss of mutant animals as a consequence of prolonged suffering from severe albuminuria and/or nephropathy. Screening mice for albuminuria at an increased age might lead to a higher level of penetrance as well as to additional albuminuric phenotypes due to alternative ENU-induced mutations.

Propagation of the mutant lines as well as the examination of the genetic cause of the aberrant phenotype are facilitated by a high phenotypic penetrance. The phenotypic analysis of the mutation may be improved by analyzing the animals more frequently, by examining mice with an older age and/or by searching for additional deviations in the clinical chemistry parameters which are indicative of the mutation. The standard use of albuminspecific antibodies for the increase of the detection sensitivity contrasts with the high throughput nature of the established test. Susceptibility to renal damage was found to be dependent on the genetic background $[15,16]$ [http://www.jax.org/phenome; http://www.eumorphia. org]. Therefore, generation and subsequent analysis of hybrids and/or congenic strains may also influence the phenotypic penetrance.

The strategy for the analysis of the number and the chromosomal position of the causative dominant muta- tions in ENU projects consists in the establishment and examination of backcross matings. Briefly, $\mathrm{C} 3 \mathrm{H}$ phenotypic mutants harboring the dominant mutation are bred to wild-type mice of a second inbred strain, the resulting hybrid offspring are analyzed for the abnormal phenotype of the parent and hybrid phenotypic mutants are backcrossed to wild-type mice of the second inbred strain. The resulting backcross offspring are again analyzed for the abnormal phenotype of the parent thereby dividing them into mutant and non-mutant animals. Genomewide microsatellite linkage analysis of the DNA samples subsequently reveals the chromosomal position of the mutation, which is further analyzed by additional molecular genetic techniques. Analysis of mutant lines showing incomplete penetrance of the abnormal phenotype requires the production and examination of a great number of offspring. Therefore, due to their low phenotypic penetrance, the two mutant lines established in the primary albuminuria screen may provide candidate regions for the renal research rather than distinct candidate genes.

Most mouse models which have been produced to date by genetic engineering techniques (additive gene transfer, gene knockout) show a lack of relevant phenotypes for their subsequent use in the analysis of the genetic factors which are involved in chronic kidney diseases $[15,17]$. Due to the triggering of random mutations by ENU, the phenotype-driven method has the impact to provide novel kidney disease models $[18,19]$.

In summary, we showed the selection of mutant mice according to the symptom albuminuria and the inheritance of the aberrant phenotype to subsequent generations. In addition, the screen was established for the assessment of the involvement of kidney lesions to other mutant phenotypes prior to the detailed pathohistologic analysis. Both the mapping and subsequent identification of the causative mutation and the precise pathological analysis of the mutation will establish unique models for nephropathies.

\section{Acknowledgements}

This work was supported by the German Human Genome Project (DHGP) (B.R., T.V.T.) and the National Genome Research Network (NGFN) (M.K.). 


\section{References}

1 Brown SD, Balling R: Systematic approaches to mouse mutagenesis. Curr Opin Genet Dev 2001;11:268-273.

2 Silver LM: Mouse Genetics: Concepts and Applications. New York, Oxford University Press, 1995.

3 Russ A, Stumm G, Augustin M, Sedlmeier R, Wattler S, Nehls M: Random mutagenesis in the mouse as a tool in drug discovery. Drug Discov Today 2002; 7:1175-1183.

4 Hrabe de Angelis MH, Flaswinkel H, Fuchs H, Rathkolb B, Soewarto D, Marschall S, Heffner S, Pargent W, Wuensch K, Jung M, Reis A, Richter T, Alessandrini F, Jakob T, Fuchs E, Kolb H, Kremmer E, Schaeble K, Rollinski B, Roscher A, Peters C, Meitinger T, Strom T, Steckler T, Holsboer F, Klopstock T, Gekeler F, Schindewolf C, Jung T, Avraham K, Behrendt H, Ring J, Zimmer A, Schughart K, Pfeffer K, Wolf E, Balling R: Genome-wide, largescale production of mutant mice by ENU mutagenesis. Nat Genet 2000;25:444-447.

5 Rathkolb B, Decker T, Fuchs E, Soewarto D, Fella C, Heffner S, Pargent W, Wanke R, Balling R, Hrabe de Angelis M, Kolb HJ, Wolf E: The clinical-chemical screen in the Munich ENU Mouse Mutagenesis Project: Screening for clinically relevant phenotypes. Mamm Genome 2000;11:543-546.
6 Rathkolb B, Fuchs E, Kolb HJ, Renner-Muller I, Krebs O, Balling R, Hrabe de Angelis M, Wolf E: Large-scale N-ethyl-N-nitrosourea mutagenesis of mice - From phenotypes to genes. Exp Physiol 2000;85:635-644.

7 Saborio P, Scheinman J: Genetic renal disease. Curr Opin Pediatr 1998;10:174-183.

8 D'Amico G, Bazzi C: Pathophysiology of proteinuria. Kidney Int 2003;63:809-825.

9 Klahr S, Morrissey J: Progression of chronic renal disease. Am J Kidney Dis 2003;41:S3S7.

10 Tryggvason K, Pettersson E: Causes and consequences of proteinuria: The kidney filtration barrier and progressive renal failure. J Intern Med 2003;254:216-224.

11 Beynon RJ, Hurst JL: Multiple roles of major urinary proteins in the house mouse, $\mathrm{Mus}$ domesticus. Biochem Soc Trans 2003;31:142146.

12 Loeb WF, Quimby FW: The Clinical Chemistry of Laboratory Animals. Philadelphia, Taylor \& Francis, 1999.

13 Doi T, Striker LJ, Gibson CC, Agodoa LY, Brinster RL, Striker GE: Glomerular lesions in mice transgenic for growth hormone and insulin-like growth factor-I. I. Relationship between increased glomerular size and mesangial sclerosis. Am J Pathol 1990;137:541-552.
14 Wolf E, Wanke R: Growth hormone overproduction in transgenic mice: Phenotypic alterations and deduced animal models; in van Zutphen LFM, van der Meer M (eds): Welfare of Transgenic Animals. Heidelberg, Springer, 1997, pp 26-47.

15 Anders H, Schlondorff D: Murine models of renal disease: Possibilities and problems in studies using mutant mice. Exp Nephrol 2000; 8:181-193.

16 Paigen K, Eppig JT: A mouse phenome project. Mamm Genome 2000;11:715-717.

17 Terzi F, Burtin M, Friedlander G: Using transgenic mice to analyze the mechanisms of progression of chronic renal failure. J Am Soc Nephrol 2000;11(suppl 16):144-148.

18 Korstanje R, DiPetrillo K: Unraveling the genetics of chronic kidney disease using animal models. Am J Physiol Renal Physiol 2004;287: F347-F352.

19 Sadl V, Jin F, Yu J, Cui S, Holmyard D, Quaggin S, Barsh $\mathrm{G}$, Cordes S: The mouse Kreisler (Krml1/MafB) segmentation gene is required for differentiation of glomerular visceral epithelial cells. Dev Biol 2002;249:16-29. 